\title{
PARETO OPTIMAL MMPROVEMENTS FOR SUNSPOTS: THE GOLDEN RULE AS A TARGET FOR STABILIZATION*
}

\author{
Subir K. Chattopadhyay**
}

WP-AD 94-19

* This paper is based on my dissertation at SUNY Stony Brook. I would liket to thank my supervisor, T.J. Muench, for his advice and encouragement. O. Galor's comments on a previous draft, and discussions with M. Kurz, J. Peck, H.M. Polemarchakis, B. Smith and I. Zilcha, are gratefully acknowledged. Comments from F. Marhuenda, two referees and, especially, M. Woodford, did much to improve the exposition. Thanks are due to the Lady Davis Foundation for supporting my stay at the Hebrew University, and the IVIE and DGICYT PB 92-0342 at Alicante, where succesive drafts were pr pared. Any remaining errors are my own.

** University of Alicante. 
Editor: Instituto Valenciano de

Investigaciones Económicas, S.A.

Primera Edición Diciembre 1994.

ISBN: 84-482-0798-X

Depósito Legal: V-4616-1994

Impreso por Copisteria Sanchis, S.L., Quart, 121-bajo, 46008-Valencia.

Printed in Spain. 


\title{
PARETO OPTIMAL IMPROVEMENTS FOR SUNSPOTS: THE GOLDEN RULE AS A TARGET FOR STABILIZATION
}

\author{
Subir K. Chattopadhyay
}

\begin{abstract}
The stationary sunspot equilibria of a simple one good OLG economy are considered. These equilibria are known to be suboptimal. We show that, for any such equilibrium allocation, there always exists a Pareto optimal improvement which has the additional property of reaching the Golden Rule in finite time, i.e., the monetary steady state acts as a target. We also show that, in general, periodic allocations cannot be used as targets. The result is interpreted as a welfare theoretical justification for stabilization policy.
\end{abstract}

KEY WORDS: Sunspot equilibria, Pareto optimal improvement, stabilization. 



\section{Introduction}

Recent results show that a simple one good overlapping generations economy (OLG) with deterministic characteristics can exhibit stationary sunspot fluctuations (see, e.g., Azariadis [1]). Following Cass and Shell [3], these are rational expectations equilibria in which uncertainty matters only because agents believe that it does even though it does not affect preferences and endowments; in addition, prices and allocations are random and depend on only the current realization of the exogenous signal. These equilibria occur in large numbers and, under a standard criterion of efficiency, they are suboptimal.

There is a growing literature dealing with the issue of "stabilizing" these sunspot equilibria (Grandmont [14], and [15], Woodford [17], and the recent Symposium in Economic Theory [9]). The objective in these papers is to specify policies which lead to a determinate and deterministic equilibrium, thus eliminating Stationary Sunspot Equilibria (SSE). ${ }^{1}$ A remarkable property of many of these policies is that, if they are announced before any trading takes place, and if they are credible, then intervention is never actually required; however, the policy induced allocation need be neither Pareto optimal nor need it improve over the SSE allocation. Hence, even though the policies in these papers have some appealing properties, like non-intervention, it is not clear that they are desirable in terms of their welfare implications.

In this paper, our focus is on allocations which are Pareto optimal improvements over the SSE allocation, and on the extent of intervention required by the policies which induce these allocations. We postulate that the economy is already in an SSE and prove the existence

\footnotetext{
${ }^{1}$ In our framework, the govermment is in a position to affect the allocations that agents receive by exercising its right to augment or tax away fiat money, or make interest payments on money balances held. A policy consists of a sequence of such taxes (subsidies) and interest rates, and generates a correponding sequence of money supplies. A policy will be said to be interventionist in a period if the money supply in that period is not the same as the money supply in the previous period.
} 
of allocations that i) Pareto improve over the expected utility from the SSE, conditional on the state in the decision period, ii) that are also Pareto optimal, and iii) that use the Golden Rule allocation as a "target" which is reached in a finite number of periods. The last property implies that these allocations can be supported as equilibria by policies which require intervention in a finite number of periods, since the Golden Rule allocation can be supported by a constant money supply.

We also show that, in general, deterministic cycles (which, like the Golden Rule, are stationary Pareto optimal allocations supported by a constant money supply) cannot play the role of targets for optimal improving allocations in a sunspot environment. In other words, for many economies, the objective of inducing an allocation which is a Pareto improvement over the SSE allocation, leads to the conclusion that no "new" deterministic fluctuations will be introduced by policy makers.

Taken together, our results imply that, for a robust class of economies, any policy that induces a Pareto optimal improvement, satisfying a minimal regularity property, over the SSE allocation, and which does not require monitoring the economy forever, must be a a policy that treats the Golden Rule allocation as a target to be reached in finite time. ${ }^{2}$ These results show a new and important property of the Golden Rule, and we interpret them as a rigorous welfare justification for "stabilization" policy.

The rest of the paper is organized as follows: Section 2 introduces the economy, its perfect foresight equilibria, and the existence and welfare properties of SSE, and concludes with a restatement of the policy problem. Some preliminary results are presented in Section 3 while our Theorem, together with an alternative optimal improvement, are presented in Section 4. In Section 5 the possibility of treating periodic allocations as targets is considered and the last section concludes.

\footnotetext{
${ }^{2}$ The regularity condition rules out allocations that are non-stationary in every subsequence of periods. It is a desirable to impose such a condition since with it the information on marginal rates of substitution (MRS) required for implementing the allocation is "finite".
} 


\section{The Economy}

\subsection{The Model $^{3}$}

Consider a simple OLG economy. Time is discrete, $t=0,1,2, \ldots$, and there is one good in each period. At each $t \geq 1$, one agent is born and lives for that and the next period. Agents are assumed to be identical across generations. Denote the consumption vector of the agent of generation $t$ by $\left(y_{t}, z_{t}\right)$ and call it an outcome. ${ }^{4}$ Let the preferences of every agent be representable by a von Neumann Morgenstern utility function $U\left[y_{t}, z_{t}\right]$ defined on $R_{+}^{2}$ and let endowments be denoted by $\left(\omega^{y}, \omega^{\circ}\right)$.

Assumption 1: $U: R_{+}^{2} \rightarrow R$ is $C^{2}$ with $D U>>0$ and $D^{2} U$ negative definite, on $R_{++}^{2}$; the closure of every indifference curve which passes through $R_{++}^{2}$ is contained in $R_{++}^{2}$.

Assumption 2: $\left(\omega^{y}, \omega^{o}\right) \in R_{++}^{2}$.

Assumptions 1 and 2 are standard technical requirements and will be treated as maintained hypotheses. Also let the agent born at $t=0$ have a monotone increasing utility function $u\left(z_{0}\right)$, and second period endowment $\omega^{\circ}$. Further assume that agent $t$ receives a lump-sum transfer (possibly negative) of fiat money from the government when old, $\tau_{t} .{ }^{5} \mathrm{~A}$ monetary policy will be given by a. sequence of transfers. If $\tau_{0}=M \neq 0, \tau_{t}=0$ for all $t=1,2,3, \ldots$, then we have a constant money supply.

\subsection{Perfect Foresight}

Under perfect foresight, where all the future prices and transfers are known with certainty, the consumers' maximization problems are:

Agent 0: $\quad \max u\left(z^{0}\right)$

$$
\text { s.t. } \quad p_{1} z^{0}=p_{1} \omega^{0}+\tau^{0} \quad z^{0} \geq 0
$$

Agent $t: \quad \max U\left[y_{t}, z_{t}\right]$

$$
\text { s.t. } \quad p_{t} y_{t}+p_{t+1} z_{t}=p_{t} \omega^{y}+p_{t+1} \omega^{\circ}+\tau_{t} \quad y_{t} \geq 0 \quad z_{t} \geq 0 .
$$

\footnotetext{
${ }^{3}$ We use the OLG model; since most of the results that we use are well known in the literature, we refrain from giving citations. For details see, e.g., Geanakoplos [11] and Geanakoplos and Polemarchakis [12]. The latter reference gives a detailed bibliography.

${ }^{4}$ Notation used: for cleterministic variables, subscripts indicate the generation that we are referring to, and, in the case of prices, they identify the period.

${ }^{5}$ We do not allow the government to purchases goods, to account for unmodeled public goods, or to levy distortionary taxes.
} 
A sequence of prices such that agents optimize and markets clear at each date,

$$
y_{t+1}+z_{t}=\omega^{y}+\omega^{\circ} \quad \forall \quad t=0,1,2,3, \ldots,
$$

will be called a perfect foresight equilibrium (PFE) sequence.

Under Assumptions 1 and 2, PFE exist; in particular, there exists a monetary steady state (the Golden Rule). The Golden Rule is the allocation that gives maximal utility to the representative agent in the class of stationary allocations; $\left(y^{G R}, z^{G R}\right)$ will denote the Golden Rule outcome. Note that the MRS at the Golden Rule is one.

Remark 1: In this economy, any feasible allocation can be supported as a PFE by some sequence of prices and transfers; in particular, the Second Welfare Theorem holds.

\subsection{Stationary Sunspot Equilibria ${ }^{6}$}

\subsubsection{Existence}

The SSE that we consider are based on a time-homogeneous Markov process with two states, $s \in\{\alpha, \beta\}$. The transition matrix for the process is given by

$$
\Pi=\left(\begin{array}{cc}
\pi^{\alpha \alpha} & 1-\pi^{\alpha \alpha} \\
1-\pi^{\beta \beta} & \pi^{\beta \beta}
\end{array}\right)
$$

where $0 \leq \pi^{\alpha \alpha} \leq 1,0 \leq \pi^{\beta \beta} \leq 1$, and $\pi^{s s^{\prime}}=1-\pi^{s s}$, is the probability of being in state $s^{\prime}$ in the next period conditional on being in state $s$ in the current period. The realization of this random variable does not influence any of the real fundamentals of the economy so that preferences and endowments continue to be deterministic. But agents, nonetheless, believe that this random variable does affect prices. They form one point conditional distributions of the prices in each state and behave so as to maximize expected utility using their knowledge of the current state and the transition matrix. An SSE is a pair of prices and associated allocations such that agents beliefs about prices are fulfilled and their optimizing demands clear markets. Stationarity of the equilibrium refers to the fact that the date of the period is irrelevant in determining prices, only the current state of nature matters.

Given today's state, $s$, the agents act so as to ${ }^{7}$

$$
\begin{aligned}
& \max _{y^{s}, z^{s \alpha}, z^{s \beta}, m_{d}^{s}} \pi^{s \alpha} U\left[y^{s}, z^{s \alpha}\right]+\pi^{s \beta} U\left[y^{s}, z^{s \beta}\right], \quad s=\alpha, \beta \\
& \text { s.t. } \quad p^{s} y^{s}+m_{d}^{s}=p^{s} \omega^{y}, \quad p^{\alpha} z^{s \alpha}=p^{\alpha} \omega^{o}+m_{d}^{s}, \quad p^{\beta} z^{s \beta}=p^{\beta} \omega^{o}+m_{d}^{s}
\end{aligned}
$$

\footnotetext{
${ }^{6}$ Guesnerie and Woodford [15] provide a comprehensive survey of the burgeoning literature dealing with endogenous fluctuations (of which sunspots are a special case). For a more technical treatment of results on existence and the properties of the equilibrium set, see Chiappori and Guesnerie [7].

${ }^{7}$ Notation nsed: for stationary stochastic variables, superscripts represent the state.
} 
Thus the agent must meet her budget constraint in each state separately, indicating the lack of contingent claims markets imposed by the structure of generational overlap. Income can be transferred across time only by using money which is a "nominal" asset.

We assume that the money supply is constant. Goods market clearing requires that

$$
y^{s}+z^{\alpha s}=\omega^{y}+\omega^{o}=y^{s}+z^{\beta s}, \quad s=\alpha, \beta .
$$

Markets clear in each state, with the implication that $z^{\alpha s}=z^{\beta s} \stackrel{\text { def }}{=} z^{s}, s=\alpha, \beta$.

Definition 1: Given preferences, initial endowments and a constant money supply $M \neq 0$, the six-tuple $\left(p^{\alpha}, p^{\beta} ; y^{\alpha}, y^{\beta}, z^{\alpha}, z^{\beta}\right)$ will constitute an SSE for $\Pi$ if:

(i) the demands expressed by the agents at those prices clear the markets;

(ii) $y^{\alpha} \neq y^{\beta}$ and;

(iii) $\quad I I \neq\left(\begin{array}{ll}0 & 1 \\ 1 & 0\end{array}\right)$.

The second restriction rules out a trivial sort of SSE an example of which would be the Golden Rule. In effect, it prevents the identity matrix from generating SSE. The third condition rules out deterministic cycles of period two from being labelled as SSE. We note that sufficient conditions for the existence of SSE are well known (see, e.g., Azariadis [1]).

\subsubsection{Welfare Properties}

Turning to the welfare properties of SSE we note that various optimality criteria have been proposed for OLG economies with uncertainty because the demographic structure endogenously generates market incompleteness. We propose to use the weakest criterion which renders SSE inefficient (see Cass and Shell [3], Dutta and Polemarchakis [8], and Peck [16]); this ensures that the problem which we wish to treat is non-vacuous. Our criterion, which is of the ex-ante sort and in the spirit of the atemporal Arrow-Debreu economy, treats agents born in different sunspot states as identical (recall that preferences and endowments are unaffected by the state of birth). We note that with a weaker criterion (for example, one which treats the agents born in different sunspot states as distinct individuals) the SSE allocation will turn out to be efficient.

Given the intertemporal nature of the economy, we need to specify a starting point from which point onwards welfare comparisons will be made; without loss of generality we shall label this point as period one, though it could be any arbitrary period. Also, we refer to 
stochastic allocations in our definitions without defining them formally; it suffices to take them to be contingent on the sunspot process (see Remark 2). ${ }^{8}$

We need some notation first. Let $\Pi_{t} \stackrel{\text { def }}{=}\left(\Pi^{\prime}\right)^{t-1}$ be the matrix which gives the probability, $\pi_{t}^{s s^{\prime}}$, of being in state $s^{\prime}$ in period $t$ given that the economy started in state $s$ in period 1, i.e., the conditional probability distribution.

Consider a particular stochastic allocation $\left\{\left(y_{t}, z_{t}\right)\right\}_{t=1}^{t=+\infty}$. We can define the sequence of conditional ex-ante expected utility levels,

$$
E U\left[\left\{\left(y_{t}, z_{t}\right)\right\}_{t=1}^{t=+\infty}\right] \stackrel{\text { def }}{=}\left\{E U_{j}\left[\left\{\left(y_{t}, z_{t}\right)\right\}_{t=1}^{t=+\infty}\right]\right\}_{j=1}^{j=+\infty}
$$

from this allocation where $E U_{j}\left[\left\{\left(y_{t}, z_{t}\right)\right\}_{t=1}^{t=+\infty}\right]$ is the expected utility of the agent born in period $j$ where the expectation is taken relative to the conditional distribution (conditional on information available at $t=1$ ) generated by the process on which the allocation under consideration is contingent. The stochastic sequence corresponding to the SSE allocation will be denoted by $s($.$) , while the sequence of ex-ante expected utility levels from the SSE$ will be denoted by $E U[s()$.$] where$

$$
E U_{t}[s(.)]=\pi_{t}^{s \alpha}\left\{\pi^{\alpha \alpha} U\left[y^{\alpha}, z^{\alpha}\right]+\pi^{\alpha \beta} U\left[y^{\alpha}, z^{\beta}\right]\right\}+\pi_{t}^{s \beta}\left\{\pi^{\beta \alpha} U\left[y^{\beta}, z^{\alpha}\right]+\pi^{\beta \beta} U\left[y^{\beta}, z^{\beta}\right]\right\} .
$$

Definition 2: A feasible allocation is a sequence of (possibly random) vectors in $R_{++}^{2}$, $\left\{\left(y_{t}, z_{t}\right)\right\}_{t=1}^{t=+\infty}$, and a scalar, $z_{0} \in R_{++}$, such that $y_{t}+z_{t-1}=\omega^{y}+\omega^{o} \quad$ for each realization and $\forall t=1,2,3, \ldots$

Definition 3: A feasible allocation $\left\{\left(y_{t}, z_{t}\right)\right\}_{t=1}^{t=+\infty}$ and $z_{0}$ dominates the feasible allocation $\left\{\left(\tilde{y}_{t}, \tilde{z}_{t}\right)\right\}_{t=1}^{t=+\infty}$ and $\tilde{z}_{0}$, if $u\left(z_{0}\right) \geq u\left(\tilde{z}_{0}\right)$ and $E U_{j}\left[\left\{\left(y_{t}, z_{t}\right)\right\}_{t=1}^{t=+\infty}\right] \geq E U_{j}\left[\left\{\left(\tilde{y}_{t}, \tilde{z}_{t}\right)\right\}_{t=1}^{t=+\infty}\right]$ $\forall j=1,2,3, \ldots$ with strict inequality for at least one $j$ or agent 0 .

Definition 4: A feasible allocation, $\left\{\left(y_{t}, z_{t}\right)\right\}_{t=1}^{t=+\infty}$ and $z_{0}$, is Pareto optimal if there is no other feasible allocation, $\left\{\left(\tilde{y}_{t}, \tilde{z}_{t}\right)\right\}_{t=1}^{t=+\infty}$ and $\tilde{z}_{0}$, which dominates it.

We now prove the suboptimality of SSE following the proof in Cass and Shell [3] and Peck [16]. Consider a given SSE and define the Expected allocation by

$$
\bar{z}_{0} \stackrel{\text { def }}{=} z^{s} ; \quad \bar{y}_{t} \stackrel{\text { def }}{=} \pi_{t}^{s \alpha} y^{\alpha}+\pi_{t}^{s \beta} y^{\beta} ; \quad \bar{z}_{t} \stackrel{\text { def }}{=} \pi_{t+1}^{s \alpha} z^{\alpha}+\pi_{t+1}^{s \beta} z^{\beta} ; \quad \forall t=1,2,3, \ldots,
$$

where $\pi_{1}^{s s^{\prime}}=0$ or 1 as the state in period one is known. Note that the Expected allocation is a function of the state in period one, i.e., "history" plays a role in this analysis.

\footnotetext{
${ }^{8}$ Deterministic allocations are, as usual, special cases of stochastic allocations.
} 
Proposition 1: If $s($.$) is an SSE allocation then it is not Pareto optimal.$

Proof: Market clearing implies that $y^{s}=-z^{s}+\omega^{y}+\omega^{o}$ for $s=\alpha, \beta$. Hence, $\bar{y}_{t}=$ $-\bar{z}_{t-1}+\omega^{y}+\omega^{o}$, so that the Expected allocation, $\left\{\bar{y}_{t}, \bar{z}_{t}\right\}_{t=1}^{t=+\infty}$ and $\bar{z}_{0}$, is feasible. By A.1 (Jensen's Inequality), and (ii) and (iii) in Definition 1, it is improving.

Q.E.D.

Remark 2: In this framework, any feasible stochastic allocation can be dominated by the corresponding "certainty" or "expected" allocation, as Proposition 1 clearly shows, so it can never be Pareto optimal. Hence, the search for Pareto optimal allocations can safely be restricted to the class of certainty allocations.

\subsection{The Policy Problem}

We shall assume that the economy under consideration is at an SSE. By Proposition 1, such an allocation is not optimal and one could easily provide technical conditions under which an optimal improvement exists. This would resolve the existence problem (by Remark 1, also the policy problem) on paper; however, it would not tell us much about the characteristics of what the government would have to do.

We approach the problem with a different perspective. Our goal is to show the existence of a particular class of Pareto optimal improvements for which the Golden Rule acts as a target which is reached in finite time. This lets us justify stabilization policy making on a solid welfare theoretical footing.

\section{Preliminary Results}

In this section we present some preliminary results. Given an SSE, we characterize the long-run behaviour of the expected utility levels conditional on the state in period one, as well as the behaviour of the Expected allocation defined in Subsection 2.3.2.

We begin by showing that the sequence of probabilities defined in Subsection 2.3.2 converges to a limit stationary distribution which is independent of the starting state.

Lemma 1: $\lim _{t \rightarrow+\infty} \Pi_{t}=\Pi^{*}$, i.e., it exists and it is given by

$\lim _{t \rightarrow+\infty} \pi_{t}^{s \alpha}=\pi^{* s \alpha} \stackrel{\text { def }}{=} \pi^{\alpha}=\frac{1-\pi^{\beta \beta}}{2-\pi^{\alpha \alpha}-\pi^{\beta \beta}} ; \lim _{t \rightarrow+\infty} \pi_{t}^{s \beta}=\pi^{* s \beta} \stackrel{\text { def }}{=} \pi^{\beta}=\frac{1-\pi^{\alpha \alpha}}{2-\pi^{\alpha \alpha}-\pi^{\beta \beta}}$.

Proof: The behaviour of $\Pi_{t}$, as $t$ goes to infinity, is determined by the eigenvalues of the matrix $\Pi$. Since it is a stochastic matrix, 1 is always one of the eigenvalues. Also, 
conditions (ii) and (iii) in Definition 1 immediately imply that the other eigenvalue is smaller than 1 in absolute value making II a fully regular matrix. From the Perron-Frobenius theorem, the result follows. (See Gantmacher [10] pgs. 50-66.)

The stationary distribution is easily calculated from a pair of simultaneous equations.

Q.E.D.

Since the Expected allocation and the sequence of ex-ante expected utilities from the SSE allocation (both conditional on the state in period one) are linear in the probabilities, Lemma 1 implies that:

Proposition 2: (i) $\lim _{t \rightarrow+\infty}\left(\bar{y}_{t}, \bar{z}_{t}\right) \stackrel{\text { def }}{=}\left(\bar{y}^{*}, \bar{z}^{*}\right)$;

(ii) $\bar{y}^{*}-\omega^{y}=-\left(\bar{z}^{*}-\omega^{o}\right)$;

(iii) $\lim _{t \rightarrow+\infty} E U_{t}[s()]=.\pi^{\alpha}\left\{\pi^{\alpha \alpha} U\left[y^{\alpha}, z^{\alpha}\right]+\pi^{\alpha \beta} U\left[y^{\alpha}, z^{\beta}\right]\right\}+$ $+\pi^{\beta}\left\{\pi^{\beta \alpha} U\left[y^{\beta}, z^{\alpha}\right]+\pi^{\beta \beta} U\left[y^{\beta}, z^{\beta}\right]\right\}$ $\stackrel{\text { def }}{=} E U^{*}[s()$.$] .$

Lemma 2: $U\left[\bar{y}^{*}, \bar{z}^{*}\right]>E U^{*}[s()$.$] .$

Proof: Note that both the sides of the inequality in the lemma are well defined by virtue of Proposition 2. Furthermore, the outcome $\left(\bar{y}^{*}, \bar{z}^{*}\right)$ is the expected value of the stochastic outcome on the right hand side of the inequality (the statement of Proposition 2 contains an explicit expression for $\left.E U^{*}[s()].\right)$. The result follows from Jensen's Inequality. Q.E.D.

These two observations imply that for some large $N$ the Golden Rule outcome is always preferred by all succeeding generations to the conditional expected utility from the SSE allocation.

Proposition 3: There exists an $N$ such that $U\left[y^{G R}, z^{G R}\right]>E U_{t}[s().] \quad \forall \quad t>N$; in particular, $U\left[y^{G R}, z^{G R}\right]>E U^{*}[s()$.$] .$

Proof: The definition of the Golden Rule allocation implies that $U\left[y^{G R}, z^{G R}\right] \geq U\left[\bar{y}^{*}, \bar{z}^{*}\right]$. Also, the continuity of $U$ and Proposition 2 imply that $U\left[\bar{y}_{t}, \bar{z}_{t}\right]$ converges to the right hand side of this inequality. The result follows directly from Lemma 2 and the continuity of $E U_{t}[s()$.$] in \pi_{t}^{s \alpha}$ and $\pi_{t}^{s \beta}$.

Q.E.D.

Remark 3: Note that $N$ could be zero. However, $N$ need not be uniformly bounded unless Assumptions 1 and 2 are strengthened to restrict the class of economies. 


\section{Optimal Improving Allocations}

In this section we present our Theorem. But first we consider the possibility of using the Expected allocation as a Pareto optimal improvement over the SSE allocation. The section ends with a comparison of the policies that support the two allocations.

Proposition 4: The Expected allocation, $\left\{\left(\bar{y}_{t}, \bar{z}_{t}\right)\right\}_{t=1}^{t=+\infty}$ and $\bar{z}_{0}$, is a Pareto optimal improvement over the SSE allocation if $\bar{y}^{*}<y^{G R}$.

Proof: From Proposition 1, the Expected allocation is a feasible improvement; therefore, from Remark 1, it can be supported as a PFE. Also, from Proposition 2 (ii), the limit of the Expected allocation is a feasible stationary allocation. Thus, $U_{1}\left[\bar{y}^{*}, \bar{z}^{*}\right] / U_{2}\left[\bar{y}^{*}, \bar{z}^{*}\right]>1$, since $\bar{y}^{*}<y^{G R}$, by hypothesis, and by Assumption 1 (strict convexity of preferences and smoothness). Now, using the convergence properties asserted in Proposition 2 (i), we can easily show that the sequence of supporting prices must converge to 0 , and optimality of the allocation follows from an application of the Cass criterion (see [12]). Q.E.D.

We now state our main result. We prove the existence of an allocation which is always Pareto improving and Pareto optimal, and which has a very simple and appealing structure after a finite number of periods, namely, that of the Golden Rule.

Theorem: The Finite Intervention allocation, $\left\{\left(\widehat{y}_{t}, \widehat{z}_{t}\right)\right\}_{t=1}^{t=+\infty}$ and $\widehat{z}_{0}$, constructed below, is well-defined, is always a Pareto optimal improvement over the SSE allocation, and has the property that it reaches the Golden Rule allocation in a finite number of periods.

Proof: Let $N$ be the finite number defined precisely in Proposition 3 . Consider the allocation given as follows:

$$
\widehat{z}_{0}=\bar{z}_{0}, \quad\left(\widehat{y}_{t}, \widehat{z}_{t}\right)=\left(\bar{y}_{t}, \bar{z}_{t}\right), \quad \forall t=1, \ldots, N,
$$

(i) and if $U\left[\bar{y}_{N+1}, z^{G R}\right] \geq E U_{N+1}[s()$.$] (in particular, when \bar{y}_{N+1} \geq y^{G R}$ ), then

$$
\left(\widehat{y}_{N+1}, \widehat{z}_{N+1}\right)=\left(\bar{y}_{N+1}, z^{G R}\right)
$$

and

$$
\left(\widehat{y}_{t}, \widehat{z}_{t}\right)=\left(y^{G R}, z^{G R}\right), \quad \forall t>N+1
$$

(ii) otherwise, let

$$
\widehat{y}_{N+1}=\bar{y}_{N+1} \text {, }
$$

and define

$$
\widehat{z}_{N+1} \quad \text { by } \quad U\left[\widehat{y}_{N+1}, \widehat{z}_{N+1}\right]=E U_{N+1}[s(.)]
$$

let

$$
\widehat{y}_{N+2}=-\widehat{z}_{N+1}+\omega^{y}+\omega^{o} \text {, }
$$

and define

$$
\widehat{z}_{N+2} \quad \text { by } U\left[\widehat{y}_{N+2}, \widehat{z}_{N+2}\right]=E U_{N+2}[s(.)] ;
$$


etc., (so we maintain feasibility, make sure that no generation is worse off, and pass on the surplus to the next generation) until, for some $\tilde{N}, U\left[\widehat{y}_{\tilde{N}}, z^{G R}\right] \geq E U_{\tilde{N}}[s()$.$] , after which,$ as in case (i), $\left(\widehat{y}_{t}, \widehat{z}_{t}\right)=\left(y^{G R}, z^{G R}\right)$ for all $t \geq \tilde{N}+1$.

Now note that, under Assumptions 1 and 2, all the SSE outcomes lie in $R_{++}^{2}$ so that the closure of the set of outcomes corresponding to the utility level $E U_{t}[s()$.$] lies in R_{++}^{2}$ for all $t$. This shows that the Finite Intervention allocation is well defined (see case (ii) in the definition of the allocation where one has to find an outcome in the set of outcomes which are certainty equivalent to $E U_{t}[s()$.$\left.] for t=N+1, \ldots, \tilde{N}\right)$.

That the proposed allocation is Pareto improving follows from Proposition 1 and the method of construction; optimality will follow from Lemma 3 once we show that the allocation does reach the Golden Rule in a finite number of periods.

Lemma 3: A feasible allocation which reaches the Golden Rule in finite time is Pareto optimal.

Proof: Since the allocation is feasible, by Remark 1 it is supportable as a PFE. Pareto optimality follows by applying the Cass criterion and noting that the Golden Rule is reached in a finite number of periods.

Q.E.D.

We now show that the allocation does reach the Golden Rule in finite time. We start with a lemma.

Lemma 4: $\widehat{y}_{t}>\bar{y}_{t} \quad \forall t=N+2, \ldots, \tilde{N}$.

Proof: The Finite Intervention allocation has been shown to be well defined. Our proof of the lemma is by induction on $t$.

Since $\widehat{y}_{N+1}=\bar{y}_{N+1}, \quad U\left[\bar{y}_{N+1}, \bar{z}_{N+1}\right]>E U_{N+1}[s()$.$] , and as the utility function$ has been assumed to be strictly monotone, the definition of $\widehat{z}_{N+1}$ implies that $\bar{z}_{N+1}>$ $\widehat{z}_{N+1}$. Then, feasibility implies that $\widehat{y}_{N+2}>\bar{y}_{N+2}$.

Now assume the assertion of the lemma to be true in period $t$, i.e., $\widehat{y}_{t}>\bar{y}_{t}$, where $t=N+3, \ldots, \tilde{N}-1$. Then, by monotonicity, $U\left[\widehat{y}_{t}, \bar{z}_{t}\right]>U\left[\bar{y}_{t}, \bar{z}_{t}\right]$ and by Proposition 1 , $U\left[\bar{y}_{t}, \bar{z}_{t}\right]>E U_{t}[s()$.$] so that the definition of the Finite Intervention allocation implies$ that $\widehat{z}_{t}<\bar{z}_{t}$ (using strict monotonicity). But, by feasibility, $\widehat{y}_{t+1}+\widehat{z}_{t}=\bar{y}_{t+1}+\bar{z}_{t}=$ $\omega^{y}+\omega^{o}$. Hence, $\widehat{y}_{t+1}>\bar{y}_{t+1}$ and the proof is complete.

Q.E.D. 
Now, using Proposition 2 and Lemma 2, we can choose an $\epsilon$ such that $U\left[\bar{y}^{*}, \bar{z}^{*}\right]-$ $E U^{*}[s()]>.2 \epsilon$. Define the set $A_{\epsilon}^{*}$ to be

$$
A_{\epsilon}^{*} \stackrel{\text { def }}{=} U^{-1}\left(\left[E U^{*}[s(.)]-\epsilon, E U^{*}[s(.)]+\epsilon\right]\right)
$$

a closed set in $R_{++}^{2}$. By Proposition 2 , there is an $M_{1}$ such that, $\forall t>M_{1}$,

$$
E U_{t}[s(.)] \in\left[E U^{*}[s(.)]-\epsilon, E U^{*}[s(.)]+\epsilon\right] .
$$

By construction of $A_{\epsilon}^{*},\left(\bar{y}^{*}, \bar{z}^{*}\right) \notin A_{\epsilon}^{*}$. Hence, there is a closed ball $\bar{B}_{\hat{\epsilon}}^{*}$ around $\left(\bar{y}^{*}, \bar{z}^{*}\right)$ such that $A_{\epsilon}^{*} \cap \bar{B}_{\hat{\epsilon}}^{*}=\emptyset$. Again, there is an $M_{2}$ such that $\forall t>M_{2},\left(\bar{y}_{t}, \bar{z}_{t}\right) \in \bar{B}_{\hat{\epsilon}}^{*}$. Choose the larger of $M_{1}$ and $M_{2}$, and call it $M_{3}$.

For the rest of the proof of the Theorem we shall refer to Figure 1.

Lemma 4 showed that $\widehat{y}_{M_{3}}>\bar{y}_{M_{3}}$. Let $\tilde{y}$ be the smallest amount that is allocated to an agent when young among all the outcomes in $\bar{B}_{\hat{\epsilon}}^{*}$. Also let $B n d$ denote that part of the boundary of the set $A_{\epsilon}^{*}$ which corresponds to the outcomes that are most preferred in that set (see the figure). Clearly, it suffices to show that given any starting outcome in $\bar{B}_{\hat{\epsilon}}^{*}$, the Finite Intervention allocation constructed using the set Bnd in place of each of the indifference curves corresponding to $E U_{t}[s()$.$] for t>M_{3}$ (as the construction actually requires), reaches the Golden Rule in a finite number of periods.

Start at a point in the set $\bar{B}_{\hat{\epsilon}}^{*}$ by letting $\widehat{y}_{M_{3}} \geq \tilde{y}$. Since $\bar{B}_{\hat{\epsilon}}^{*} \cap B n d=\emptyset$, the corresponding consumption when old will be defined by moving downwards (by convexity); feasibility will require that we then move towards the diagonal (the set of feasible stationary allocations, denoted $F$ ) and then repeat the process (see the arrows in the figure).

We now show that these downward movements are uniformly bounded below by a positive number and this will complete the proof. So consider the two sets $G H$ and $I J$, subsets of the diagonal and the set $B n d$, respectively, where $G$ is identified with the Golden Rule outcome. Since $\left(y^{G R}, z^{G R}\right) \notin A_{\epsilon}^{*}$, the segment $J G$ is of positive length. Hence, the sets $G H$ and $I J$ are disjoint, and the the minimum vertical distance between them is positive; this distance can serve as the required lower bound. ${ }^{9}$

$$
\text { Q.E.D. }
$$

\footnotetext{
${ }^{9}$ The position of the sets in the figure is without loss of generality; if the set $\bar{B}_{\hat{\varepsilon}}^{*}$ lies below the Golden Rule (relative to the diagonal), a much more direct argument can be given.
} 
FIGURE 1

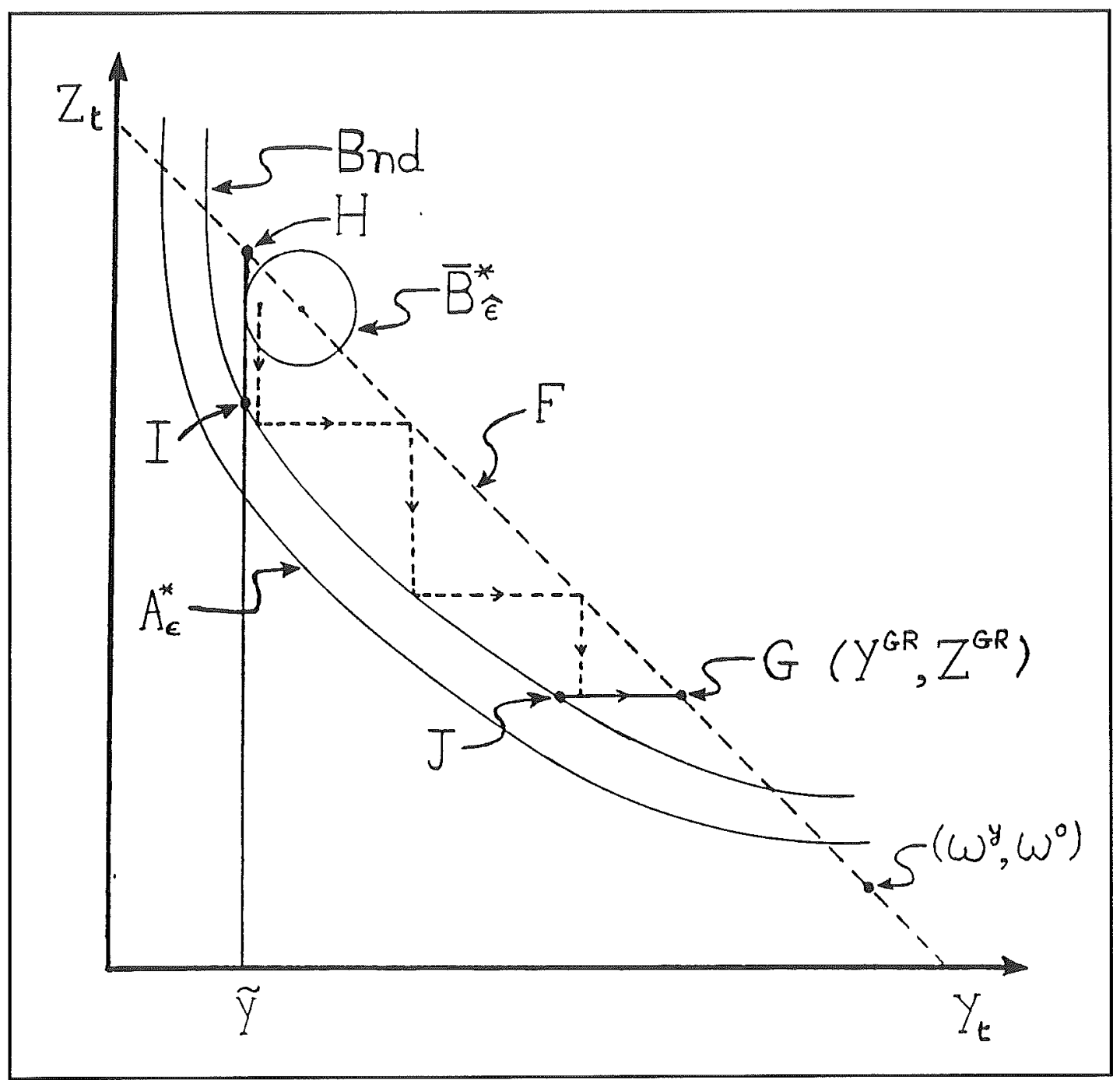


Remark 4: It should be clear from the construction that $N$ can be replaced by any $\widehat{N}>N$ and the Theorem will continue to be true; also, one could obtain a strict Pareto improvement over the SSE and still reach the Golden Rule in finite time. This implies that we have a continuum of alternatives to choose from.

Remark 5: Note that both the allocations considered in this section are robust in the sense that small changes in the transition matrix and other characteristics of the economy would still leave their potency (as optimal improvements) unaffected since they lead to strictly superior allocations all of the time (Remark 4 applies to the Finite allocation).

Remark 6: Note that the results of this section do not depend on the economy being of the Samuelson/Classical type. The sunspot literature, however, has concentrated on the Samuelson, or "positive" money, case.

We now consider the kind of intervention required in order to support these allocations. From Remark 1, we know that there exists a sequence of monetary transfers and a sequence of prices (with one nominal degree of freedom) which will support a given Pareto optimal allocation as a PFE. Hence, the implementation problem is trivial subject to the usual caveat of finding the correct transfers and prices. But it is instructive to compare these supporting prices and transfers across the results proved.

It is easy to show that in order to support the Expected allocation, when it is an optimal improvement, the govermment has to siphon money out of the system (for large $t$ ) and the nominal money supply and the nominal price level converge to zero (see Chattopadhyay [4] (Section 4.1) for the details). So, unless the government keeps changing the units in which its currency is denominated, there is the risk of making a small mistake in calculating the transfers. This would destroy the optimality of the allocation. The Finite Intervention allocation does not suffer from this defect. Since the Golden Rule is reached in finite time, there comes a time after which the money supply and the price level are constant.

Also, implementation of the Expected allocation requires knowledge of an infinite number of marginal rates of substitution while, for the Finite Intervention allocation, beyond a point, all the marginal rates of substitution are one. Since, the number $N$ could well be small (see Remark 3), this could amount to a quite a reduction in the informational requirements as far as implementation is concerned. 


\section{Cycles as "Targets"}

In the previous section we saw that the Golden Rule allocation can act as a target for optimal improvements and one might ask whether there are any other allocations, with minimal regularity properties like stationarity, which could perform the same task. Since periodic allocations (see Grandmont [13]) are Pareto optimal, and supportable by a constant money supply, it is natural to conjecture the existence of an allocation which Pareto dominates the SSE allocation, and which converges to a periodic allocation.

We now give a robust example of an economy for which the above conjecture is false. This result, when combined with the Theorem, shows that, for a robust class of economies, the only allocations which are (i) Pareto optimal improvements over the SSE allocation, (ii) do not require perpetual intervention and (iii) satisfy a minimal regularity property, are ones that converge to the Golden Rule in finite time.

So, consider an economy in which agents are relatively well endowed when young (Samuelson case), and consumption when old is a normal good. ${ }^{10}$ This puts us in the framework used in Azariadis and Guesnerie [2] and Grandmont [13].

Let $(\dot{y}, \dot{z})$ and $(\ddot{y}, \ddot{z})$ be the outcomes at a two period cycle, and say that if:

(i) $\operatorname{Min}\{\dot{y}, \ddot{y}\}<\left\{y^{\alpha}, y^{\beta}\right\}<\operatorname{Max}\{\dot{y}, \ddot{y}\}$;

(ii) $\operatorname{Min}\{\dot{z}, \ddot{z}\}<\left\{z^{\alpha}, z^{\beta}\right\}<\operatorname{Max}\{\dot{z}, \ddot{z}\}$

then the cycle is "outside" the SSE. Now, careful inspection of Theorem 4 in [2] tells us that if an SSE exists, then a two perior cycle will always exist "outside" the SSE. But notice that this implies that all the sunspot outcomes, viewed individually as certain outcomes, are strictly preferred over the worse of the two cyclic outcomes. Therefore, the conditional expected utility from the SSE is always greater than the utility from the periodic allocation at one of the periodic points. Hence, this two period cycle could not possibly provide an optimal improvement.

Now consider an economy for which an SSE exists, and for which there is: (i) a unique two period cycle, which, by the observation above, must lie outside the SSE; (ii) the economy has no other periodic equilibria. That such an economy exists can be seen as follows: as noted above, we are now in the framework of [13] and the "backward perfect foresight"

\footnotetext{
${ }^{10}$ Note that without the normality assumption, there might be no two period cycle which would be a candidate for a Pareto improvement even though an SSE does exist (see Chattopadhyay and Muench [6]). There might, however, be other periodic allocations.
} 
dynamics are well defined. By Sarkovski's Theorem, for these dynamics and a parameterized set of economies, cycles of period two will appear first. It suffices to consider an economy corresponding to a parameter value greater than the bifurcation value at which a two cycle obtains, and less than the bifurcation value at which a four cycle obtains. We have:

Proposition 5: There exist robust examples of economies with SSE and periodic PFE for which no periodic equilibrium can be a candidate for a Pareto improvement.

Remark 7: Note that there are robust examples of economies for which a periodic PFE is an optimal improvement. To see this, note that for a given SSE the limiting expected utility is bounded away from the Golden Rule utility level (by Proposition 3). Now modify the preferences around the Golden Rule so as to generate a two period cycle with outcomes uniformly close to the Golden Rule. This is a local perturbation and does not jeoperdize the existence of the SSE. Such a cycle can clearly serve as a target for an optimal improvement.

\section{Concluding Comments}

We considered a model in which the economy is locked into an inefficient SSE and then solved the planner's problem of designing policies leading to optimal improvements with full information about the economy. ${ }^{11}$ We showed the existence of a (continuum) of policies which achieve this goal and reach the Golden Rule in finite time so that policy intervention is required for only a finite number of periods. So attempts to neutralize the effect of the sunspot variable lead, in a very natural sense, to an effort to "stabilize" the economy around a stationary allocation with some very nice welfare properties. Our results indicate that stabilization policy could in fact have a rigorous welfare theoretical foundation.

In terms of generalizations, we note that Lemma 1 is the key to all our results. This result will continue to hold in more general settings, so long as the process is "mixing." Therefore, our results extend immediately to the case with more than two sunspot states or more than one type of agent. We conjecture that an analogue of the Theorem can be proved for models with more than one good in each period and also heterogeneity among agents (for an appropriate generalization of the equilibrium concept) so long as agents are assumed to be risk averse. However, the informational requirements for implementing such allocations will be very high.

\footnotetext{
${ }^{11}$ In Chattopadhyay [5] we consider the case in which the planner does not have full information.
} 



\section{References}

[1] Azariadis, C.: Self-Fulfilling Prophecies. Journal of Economic Theory 25, 380-396 (1981)

[2] Azariadis, C., Guesnerie, R.: Sunspots and Cycles. Review of Economic Studies 53, 725-737 (1986)

[3] Cass, D., Shell, K.: Do Sunspots Matter? Journal of Political Economy 91, 189-227 (1983)

[4] Chattopadhyay, S. K.: Optimal Improvement Policies for Stationary Sunspot Equilibria in an OLG Economy: the Central Role of the Golden Rule Allocation. I.D.S. discussion paper 90-21, S.U.N.Y. Stony Brook 1990

[5] Chattopadhyay, S. K.: Information, Stabilization and Welfare: the Case of Sunspots. Working Paper No. 281, Dept. of Economics, The Hebrew University of Jerusalem 1993

[6] Chattopadhyay, S.K., Muench, T.J.: Normal Goods, Sunspots, and Cycles. Working Paper No. 282, Dept. of Economics, The Hebrew University of Jerusalem 1993

[7] Chiappori, P. A., Guesnerie, R.: Sunspot Equilibria in Market Models. In: Hildenbrand, W., Sonnenschein, H. (eds.) Handbook of Mathematical Economics, IV. New York: North Holland 1991

[8] Dutta, J., Polemarchakis, H.M.: Asset Markets and Equilibrium Processes. Review of Economic Studies, 57, 229-254 (1990)

[9] Economic Theory Symposium: Determinacy of Equilibrium Under Alternative Policy Regimes. M. Woodford (ed.) Economic Theory 4, 323-416 (1994)

[10] Gantmacher, F.: Matrix Theory Vol. II. New York: Chelsea Inc. 1959

[11] Geanakoplos, J. D.: Overlapping Generations Models of General Equilibrium. In: Eatwell, J., Milgate, M., Newman, P. (eds.) The New Palgrave Dictionary of Economics: General Equilibrium. London: MacMillan 1989

[12] Geanakoplos, J. D., Polemarchakis, H. M.: Overlapping Generations. In: Hildenbrand, W., Sonnenschein, H. (eds.) Handbook of Mathematical Economics, IV. New York: North Holland 1991

[13] Grandmont, J. M.: On Endogenous Competitive Business Cycles. Econometrica, 53, 995-1045 (1985)

[14] Grandmont, J. M.: Stabilizing Competitive Business Cycles. Journal of Economic Theory, 40, 57-76 (1986) 
[15] Guesnerie, R., Woodford, M.: Endogenous Fluctuations. In Laffont, J.J. (ed) Advances in Economic Theory, II. Cambridge: Cambridge University Press (1992).

[16] Peck, J.: On The Existence of Sunspot Equilibria in an Overlapping Generations Model. Journal of Economic Theory, 44, 19-42 (1988)

[17] Woodford, M.: Stationary Sunspot Equilibria in a Finance Constrained Economy. Journal of Economic Theory, 40, 128-137 (1986) 


\section{PUIBLISHICD ISSUES}

WP-AD 90-01 "Vector Mappings with Diagonal Images"

C. Herrero, A.Villar. December 1990.

WP-AD 90-02 "Langrangean Conditions for General Optimization Problems with Applications to Consumer Problems"

J.M. Gutierrez, C. Herrero. December 1990.

WP-AD 90-03 "Doubly Implementing the Ratio Correspondence with a 'Natural' Mechanism"

L.C. Corchón, S. Wilkie. December 1990.

WP-AD 90-04 "Monopoly Experimentation"

L. Samuelson, L.S. Mirman, A. Urbano. December 1990.

WP-AD 90-05 "Monopolistic Competition: Equilibrium and Optimality"

L.C. Corchón. December 1990.

WP-AD 91-01 "A Characterization of Acyclic Preferences on Countable Sets"

C. Herrero, B. Subiza. May 1991.

WP-AD 91-02 "First-Best, Second-Best and Principal-Agent Problems"

J. Lopez-Cuñat, J.A. Silva. May 1991.

WP-AD 91-03 "Market Equilibrium with Nonconvex Technologies"

A. Villar. May 1991.

WP-AD 91-04 "A Note on Tax Evasion"

L.C. Corchón. June 1991.

WP-AD 91-05 "Oligopolistic Competition Among Groups"

L.C. Corchón. June 1991.

WP-AD 91-06 "Mixed Pricing in Oligopoly with Consumer Switching Costs"

A.J. Padilla. June 1991.

WP-AD 91-07 "Duopoly Experimentation: Cournot and Bertrand Competition"

M.D. Alepuz, A. Urbano. December 1991.

WP-AD 91-08 "Competition and Culture in the Evolution of Economic Behavior: A Simple Example"

F. Vega-Redondo. December 1991.

WP-AD 91-09 "Fixed Price and Quality Signals"

L.C. Corchón. December 1991.

WP-AD 91-10 "Technological Change and Market Structure: An Evolutionary Approach"

F. Vega-Redondo. December 1991.

WP-AD 91-11 "A 'Classical' General Equilibrium Model"

A. Villar. December 1991.

WP-AD 91-12 "Robust Implementation under Alternative Information Structures"

L.C. Corchón, I. Ortuño. December 1991. 
WP-AD 92-01 "Inspections in Models of Adverse Selection"

I. Ortuño. May 1992.

WP-AD 92-02 "A Note on the Equal-Loss Principle for Bargaining Problems"

C. Herrero, M.C. Marco. May 1992.

WP-AD 92-03 "Numerical Representation of Partial Orderings"

C. Herrero, B. Subiza. July 1992.

WP-AD 92-04 "Differentiability of the Value Function in Stochastic Models"

A.M. Gallego. July 1992.

WP-AD 92-05 "Individually Rational Equal Loss Principle for Bargaining Problems"

C. Herrero, M.C. Marco. November 1992.

WP-AD 92-06 "On the Non-Cooperative Foundations of Cooperative Bargaining"

L.C. Corchón, K. Ritzberger. November 1992.

WP-AD 92-07 "Maximal Elements of Non Necessarily Acyclic Binary Relations"

J.E. Peris, B. Subiza. December 1992.

WP-AD 92-08 "Non-Bayesian Learning Under Imprecise Perceptions"

F. Vega-Redondo. December 1992.

WP-AD 92-09 "Distribution of Income and Aggregation of Demand"

F. Marhuenda. December 1992.

WP-AD 92-10 "Multilevel Evolution in Games"

J. Canals, F. Vega-Redondo. December 1992.

WP-AD 93-01 "Introspection and Equilibrium Selection in 2x2 Matrix Games"

G. Olcina, A. Urbano. May 1993.

WP-AD 93-02 "Credible Implementation"

B. Chakravorti, L. Corchón, S. Wilkie. May 1993.

WP-AD 93-03 "A Characterization of the Extended Claim-Egalitarian Solution" M.C. Marco. May 1993.

WP-AD 93-04 "Industrial Dynamics, Path-Dependence and Technological Change"

F. Vega-Redondo. July 1993.

WP-AD 93-05 "Shaping Long-Run Expectations in Problems of Coordination"

F. Vega-Redondo. July 1993.

WP-AD 93-06 "On the Generic Impossibility of Truthful Behavior: A Simple Approach"

C. Beviá, L.C. Corchón. July 1993.

WP-AD 93-07 "Cournot Oligopoly with 'Almost' Identical Convex Costs"

N.S. Kukushkin. July 1993.

WP-AD 93-08 "Comparative Statics for Market Games: The Strong Concavity Case"

L.C. Corchón. July 1993.

WP-AD 93-09 "Numerical Representation of Acyclic Preferences"

B. Subiza. October 1993. 
WP-AD 93-10 "Dual Approaches to Utility"

M. Browning. October 1993.

WP-AD 93-11 "On the Evolution of Cooperation in General Games of Common Interest"

F. Vega-Redondo. December 1993.

WP-AD 93-12 "Divisionalization in Markets with Heterogeneous Goods"

M. González-Maestre. December 1993.

WP-AD 93-13 "Endogenous Reference Points and the Adjusted Proportional Solution for Bargaining Problems with Claims"

C. Herrero. December 1993.

WP-AD 94-01 "Equal Split Guarantee Solution in Economies with Indivisible Goods Consistency and Population Monotonicity"

C. Beviá. March 1994.

WP-AD 94-02 "Expectations, Drift and Volatility in Evolutionary Games"

F. Vega-Redondo. March 1994.

WP-AD 94-03 "Expectations, Institutions and Growth"

F. Vega-Redondo. March 1994.

WP-AD 94-04 "A Demand Function for Pseudotransitive Preferences"

J.E. Peris, B. Subiza. March 1994.

WP-AD 94-05 "Fair Allocation in a General Model with Indivisible Goods"

C. Beviá. May 1994.

WP-AD 94-06 "Honesty Versus Progressiveness in Income Tax Enforcement Problems"

F. Marhuenda, I. Ortuño-Ortín. May 1994.

WP-AD 94-07 "Existence and Efficiency of Equilibrium in Economies with Increasing Returns to Scale: An Exposition"

A. Villar. May 1994.

WP-AD 94-08 "Stability of Mixed Equilibria in Interactions Between Two Populations"

A. Vasin. May 1994.

WP-AD 94-09 "Imperfectly Competitive Markets, Trade Unions and Inflation: Do Imperfectly Competitive Markets Transmit More Inflation Than Perfectly Competitive Ones? A Theoretical Appraisal" L. Corchón. June 1994.

WP-AD 94-10 "On the Competitive Effects of Divisionalization"

L. Corchón, M. González-Maestre. June 1994.

WP-AD 94-11 "Efficient Solutions for Bargaining Problems with Claims" M.C. Marco-Gil. June 1994.

WP-AD 94-12 "Existence and Optimality of Social Equilibrium with Many Convex and Nonconvex Firms" A. Villar. July 1994.

WP-AD 94-13 "Revealed Preference Axioms for Rational Choice on Nonfinite Sets" J.E. Peris, M.C. Sánchez, B. Subiza. July 1994. 
WP-AD 94-14 "Market Learning and Price-Dispersion"

M.D. Alepuz, A. Urbano. July 1994.

WP-AD 94-15 "Bargaining with Reference Points - Bargaining with Claims: Egalitarian Solutions Reexamined"

C. Herrero. September 1994.

WP-AD 94-16 "The Importance of Fixed Costs in the Design of Trade Policies: An Exercise in the Theory of Second Best"

L. Corchón, M. González-Maestre. September 1994.

WP-AD 94-17 "Computers, Productivity and Market Structure"

L. Corchón, S. Wilkie. October 1994.

WP-AD 94-18 "Fiscal Policy Restrictions in a Monetary System: The Case of Spain" M.I. Escobedo, I. Mauleón. December 1994.

WP-AD 94-19 "Pareto Optimal Improvements for Sunspots: The Golden Rule as a Target for Stabilization" S.K. Chattopadhyay. December 1994. 